Superalloys 2012: 12 ${ }^{\text {th }}$ International Symposium on Superalloys

\title{
MOLECULAR-DYNAMICS SIMULATIONS OF MOLTEN NI-BASED SUPERALLOYS
}

\author{
Christopher Woodward ${ }^{1}$, James Lill ${ }^{2}$, Mark Asta ${ }^{3}$, Dallas R. Trinkle ${ }^{4}$
}

\author{
${ }^{1}$ Materials and Manufacturing Directorate, Air Force Research Laboratory, Wright Patterson AFB, OH, 45433-7817, USA \\ ${ }^{2}$ High Performance Technologies Inc., Wright Patterson AFB, OH, 45433-7817, USA \\ ${ }^{3}$ Department of Materials Science and Engineering, University of California, Berkeley, CA, 94720, USA \\ ${ }^{4}$ Department of Materials Science and Engineering, University of Illinois, Urbana-Champaign, Urbana, IL, 61801, USA
}

Density Functional Theory, Liquid metal, Density, Molar volume, Diffusion

\begin{abstract}
Fundamental parameters of liquid metal alloys are calculated using a first principles approach, based on Density Functional Theory (DFT), with the goal of informing models of defect formation during solidification processing. Ab-initio molecular dynamic simulations (AIMD) are applied to liquid metal alloys ranging from simple metals to Rene-N4 and CMSX-4 to predict molar volumes (density), diffusion rates and local ordering. These include elemental Ni, Ni-5.4X, Ni-20X, and Ni-10Al$2.8 \mathrm{X}(\mathrm{X}=\mathrm{W}, \mathrm{Re}$, and $\mathrm{Ta})(\mathrm{at} \%)$ alloys at 1750 and $1830 \mathrm{~K}$. Calculated kinetics and the atomic distribution in the liquids indicate that simulations of 500 atoms run for approximately 7 ps converge the time-averaged properties, including molar volume. Overall diffusion rates and molar volumes are in good agreement with available experimental measurements, though the AIMD predictions appear to systematically underestimate thermal expansion. The method is then used to predict density inversion in three additional alloys, Ni-14Al-3W, CMSX-4 and Rene-N4 for temperatures and compositions expected in the mushy zone during directional solidification. Density inversion is predicted for these three alloys based on density and density contrast in the mushy zone, the prediction and ranking of the effect is consistent with previous studies. Predictions for molar volume are compared with the recent parameterization of molar volumes using extensive measurements of binary alloys. AIMD calculations validate the underlying assumptions of these models and illustrate the bounds in alloy chemistry for applying such techniques.
\end{abstract}

\section{Introduction}

With the increasing complexity in alloy chemistry and airfoil geometry over succeeding generations of turbine engines the probability of forming processing defects (freckles) during directional solidification has increased. These calculations offer a new approach for determining the fundamental parameters required for modeling aspects of this processing path. Convective instabilities responsible for freckle defects are produced by variations in liquid-metal density with composition and temperature across the solidification zone. A systems design leading to optimal material and component performance requires a quantitative model for predicting processing regimes for defect free castings over the widest range of refractory metal compositions. Models of thermo-chemical convection in the mushy zone have shown that such instabilities occur when a Rayleigh number exceeds a critical value [1]. The Rayleigh number, $R$, is a measure of the ratio of the buoyancy force to the retarding frictional force:

\section{$\mathrm{R}=(\Delta \rho / \rho) \mathrm{gKh} / \alpha v$}

Where $\mathrm{h}$ is the height of the mushy zone, $\mathrm{K}$ is the average permeabihity, $g$ the acceleration of gravity, $\alpha$ is the thermal diffusivity, $v$ is the kinematic shear viscosity of the fluid, and $\Delta \rho / \rho$ is the density contrast. The last term is the variation in mass density between the liquid metal near the solid-liquid dendrite interface and the cooler melt at the bottom and sides walls of the mold. Verifying models of freckle formation for a specific alloy system requires accurate values for the parameters that describe this Rayleigh number. Many of these constants, such as the viscosity, permeability and density, are difficult to measure and are not well documented for the superalloys. Recently, Mukai and coworkers have developed a multicomponent parameterization for molar volumes $[\mathrm{V}(\mathrm{c}, \mathrm{T})]$ in superalloys based on extensive experimental measurements of the densities of binary liquid Ni alloys, and a few representative ternaries [2]. The current simulations offer a new way of determining such densities and an independent test of the accuracy of the model for Mukai's parameterization of $\rho(\mathrm{c}, \mathrm{T})$.

Assuming that other parameters for the Rayleigh criteria are constant across the mushy zone, the signature for freckle formation is reduced to the condition of density inversion. This occurs when the liquid metal at higher temperature has a greater density than at lower temperatures. The density contrast depends on casting temperature gradients as well as the variations in solute concentration produced by the equilibrium partitioning between solid and liquid phases. The more complex alloys, such as Rene-N4 and CMSX4, pose additional challenges for the AIMD simulations. For these alloys it is first necessary to demonstrated that the 500 atom cell AIMD simulations can give precise and reproducible densities for simulations running for less than $\sim 10 \mathrm{ps}$.

The rest of this manuscript is organized in the following manner. First we describe how the AIMD method is applied. This includes details of the electronic structure calculations, molecular dynamics and analysis of the time averaged properties. This is followed by a results and discussion section, where each derived property is reviewed in turn. First the local atomic structure and kinetics found in the simple, binary and ternary $\mathrm{Ni}$ alloys are reviewed in order to validate the convergence of the methodology. Next, the predicted molar volumes are considered and compared with available experimental data and parametric fits to experimental data. The last part of this section is where the predictions for density inversion are presented and discussed. Finally the work is reviewed in a brief summary.

\section{Method}

The properties of molten Ni-based alloys were calculated using ab-initio molecular dynamic simulations. The time evolution was based on inter-atomic forces (i.e. the Hellmann-Feynman forces) computed directly using Density Functional Theory and the commercial software VASP (Vienna ab-initio simulation 
package) developed at the Institut für Materialphysik of the Univerität Wien) [3]. Ionic positions were evolved in time using classical Nosé-Hoover dynamics for both a fixed-number, volume, and temperature (NVT) and fixed-number, pressure and temperature (NPT) ensembles. A self-consistent charge density is calculated at each time step in order to integrate the ionic trajectories in relatively small simulation cells (500 atoms) with total simulation times ranging from 5-10 ps. Correlation times were found in the range of 40-60 fs, which is taken into account for all reported thermal average quantities and error estimates. For simulations of this size a single k point, at the gamma point, was adequate to perform the reciprocal space integrations. Partial occupancy of the electronic bands was accomplished using Fermi broadening, with an electronic temperature equivalent to that of the ionic system. All calculations used a generalized gradient approximation (PW91) with a plane-wave basis cutoff of $270 \mathrm{eV}$ [4]. Initial calculations on simple, binary and ternary alloys employed ultrasoft pseudopotentials [5,6]. Time steps $(\Delta t)$ for the MD simulations were chosen at or below $\Delta t=0.003 \mathrm{ps}$ to reduce possible drift in the conserved energy of the Nosé-Hoover dynamics. Initial configurations for $\mathrm{Ni}$ and $\mathrm{Ni}-$ Al were taken from snapshots of classical molecular dynamics simulations on classical interatomic potentials [7]. The initial configurations for simulations of other chemistries were obtained by replacing $\mathrm{Ni}$ or $\mathrm{Al}$ atoms, selected at random, with the correct target atomics species. In this manuscript the number of each atomic species gives the nomenclature for the composition of an AIMD supercell. Thus for a 500 atom cell the composition $\mathrm{Ni}-20 \mathrm{Al}\left(\right.$ at $\%$ ) takes the form: $\mathrm{Ni}_{400} \mathrm{Al}_{100}$.

Initial calculations, designed to sample and test the accuracy of the Mukai parameterization, were performed on $\mathrm{Ni}, \mathrm{Al}, \mathrm{Ni}-20 \mathrm{X}$, Ni-5.4X, and Ni-10Al-2.8X (X= W, Re, Ta) alloys (at \%) at 1750 and $1830 \mathrm{~K}$. These include alloy chemistries containing refractory metal additions (i.e. Re) where experimental measurements are currently unavailable. The Nosé-Hoover equations of motion were integrated using thermostat temperatures of $\mathrm{T}=1830$ and $\mathrm{T}=1750 \mathrm{~K}$ for compositions including: $\quad \mathrm{Al}_{500}, \quad \mathrm{Ni}_{500}, \quad \mathrm{Ni}_{400} \mathrm{Al}_{100}, \quad \mathrm{Ni}_{473} \mathrm{~W}_{27}, \quad \mathrm{Ni}_{400} \mathrm{~W}_{100}$, $\mathrm{Ni}_{473} \mathrm{Re}_{27}, \quad \mathrm{Ni}_{400} \mathrm{Re}_{100}, \quad \mathrm{Ni}_{473} \mathrm{Ta}_{27}, \quad \mathrm{Ni}_{400} \mathrm{Ta}_{100}, \quad \mathrm{Ni}_{436} \mathrm{Al}_{50} \mathrm{~W}_{14}$, $\mathrm{Ni}_{436} \mathrm{Al}_{50} \mathrm{Re}_{14}$ and $\mathrm{Ni}_{436} \mathrm{Al}_{50} \mathrm{Ta}_{14}$. Three, or more, NVT simulations at each composition and temperature were performed over a range of volumes within 5\% (above and below) the equilibrium volume. Fitting the points to an equation of state yields the equilibrium volume.

Further calculations were undertaken to document possible density inversions in Ni-Al-W, Rene-N4 and CMSX4. These calculations pose additional challenges in terms of sampling a sufficiently large configurational space and simulation times to produce a reasonably accurate estimate of the liquid metal densities. To address these issues VASP (4.6.31 and more recently 5.2) was modified to integrate the molecular dynamics equations appropriate for a fixed number, pressure and temperature (nPT) ensemble. This significantly decreases the computational effort required to calculate equilibrium volumes, liquid metal diffusion rates and viscosities. Also, these calculations employed Projected Augmented Wave pseudopotentials in order to obtain a more accurate representation of the complex chemistries found in the superalloys [6,8]. In order to explore the accuracy and reproducibility of AIMD predictions on more complex alloys, a preliminary set of calculations were run on two representative compositions of Rene-N4.

\section{Results and Discussion}

The AIMD calculations yield a variety of time-averaged quantities that provide insights to the kinetics, ordering and density in the melt. The first quantity can be used to show that the simulations are of long enough duration to produce significant interdiffusion, so that the calculated thermal averages are not biased by the choice of the initial atomic configuration. Further, in order to produce a statistically realistic liquid the cell volume must be large enough so that atomic correlations decay sufficiently over the scale of the simulation cell. These convergence conditions are considered first, before reviewing the AIMD predictions for liquid metal density.

\section{Local Ordering}

The radial distribution function, $g_{\alpha \beta}(r)$, for each pair of species $(\alpha, \beta)$ in pure $\mathrm{Ni}$ and binary $\mathrm{Ni}_{400} \mathrm{X}_{100}(\mathrm{X}=\mathrm{Al}, \mathrm{W}$, and $\mathrm{Re})$ melts are shown in Figure 1. The distribution functions converge to the uncorrelated limit (e.g. unity) in less then half the diagonal distance across the simulation cell. This illustrates that the
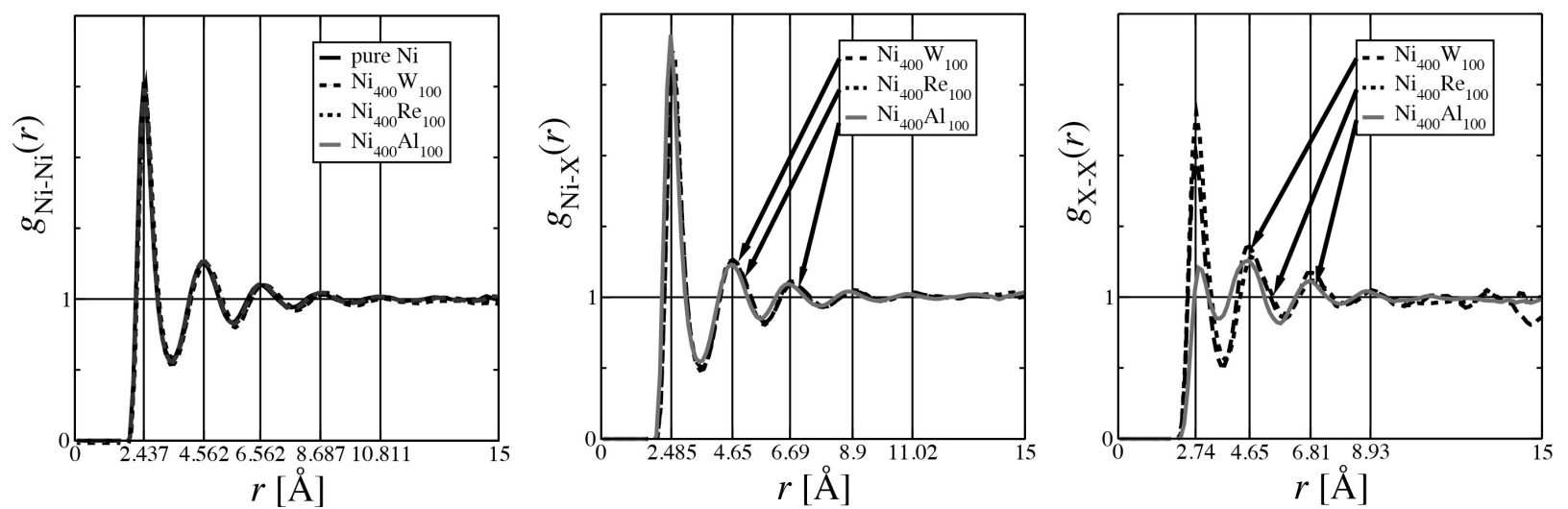

Figure 1. Radial distribution functions for Ni and Ni-20X (X=Al, W, Re) alloys at $1830 \mathrm{~K}$ for the lowest pressure NVT simulations at these chemistries. From left to right, the three plots show solvent-solvent (Ni-Ni), solvent-solute (Ni-X), and solute-solute (X-X) pairs. 
volume provide by the simulation cells is large enough to allow uncorrelated motion of individual atoms and groups of atoms. The radial distribution functions also give indications of a preferred local ordering in these binary alloys. The main $\mathrm{g}_{\mathrm{Ni}-\mathrm{X}}$ peak is larger than the solvent-solvent (Ni-Ni) and solute-solute (X-X) peaks, suggestive of a preference for chemical shortrange order between neighbors of different species. Also, the average bond length of $\mathrm{Ni}-\mathrm{Ni}$ and $\mathrm{X}-\mathrm{X}$ pairs $(\sim 2.59 \AA)$ is significantly larger than $\mathrm{Ni}-\mathrm{X}$ pairs $(\sim 2.485 \AA)$. This suggests that a chemical short range ordering where $\mathrm{Ni}$ prefers to surround itself with unlike nearest neighbors in each of the binary systems considered. Further information, beyond that available from $g(r)$, about possible short-range order and the structure of the liquids can be found through common neighbor analysis (CNA) and the angle probability distribution function $[9,10]$.

Previously we have applied these methods to Ni, Ni473-Re27 and Ni473W27 and found strong evidence for icosahedral short range ordering, with a secondary preference of bcc ordering [11]. This is consistent with experimental observations in liquid metal $\mathrm{Ni}$ and $\mathrm{Ni}-\mathrm{Ag}$ amorphous alloys [12,13] that show a preference for icosahedral ordering. This type of ordering cannot be found in crystals because it does not permit periodic filling of a 3D space, however in liquid fcc alloys it maximizes the shortrange density of the structure thus lowering the enthalpy of the system [14].

\section{$\underline{\text { Kinetics }}$}

Diffusion kinetics in these liquid metals can be estimated through the Einstein relationship using the averaged meansquare displacements. Shown in Figure 2 are the averaged mean square displacements for $\mathrm{Ni}, \mathrm{Al}, \mathrm{W}, \mathrm{Ta}$, and $\mathrm{Re}$, calculated from
Table I. Calculated diffusion parameters $\left(10^{-5} \mathrm{~cm}^{2} / \mathrm{sec}\right)$ and activation volumes Ni and Ni binary alloys at $1830 \mathrm{~K}$. Errors, representing the $95 \%$ confidence level, are derived from standard propagation of errors from the statistics of the raw data.

\begin{tabular}{|c|c|c|c|c|}
\hline \multirow{2}{*}{$\begin{array}{c}\text { Alloy } \\
\text { Composition }\end{array}$} & \multicolumn{2}{|c|}{ Solvent } & \multicolumn{2}{c|}{ Solute } \\
\cline { 2 - 5 } & $\mathrm{D}(\mathrm{Ni})$ & $\mathrm{V}_{\mathrm{a}}(\mathrm{Ni})$ & $\mathrm{D}(\mathrm{X})$ & $\mathrm{V}_{\mathrm{a}}(\mathrm{X})$ \\
\hline $\mathrm{Ni}$ & $5.3(3)$ & $1.3(3)$ & & \\
$\mathrm{Ni}-5.4 \mathrm{Re}$ & $5.0(2)$ & $1.3(2)$ & $3.6(4)$ & $0.3(5)$ \\
$\mathrm{Ni}-5.4 \mathrm{Ta}$ & $4.6(1)$ & $1.3(2)$ & $3.7(3)$ & $0.5(4)$ \\
$\mathrm{Ni}-5.4 \mathrm{~W}$ & $4.2(2)$ & $1.3(2)$ & $3.6(4)$ & $0.9(6)$ \\
$\mathrm{Ni}-20 \mathrm{Al}$ & $3.4(4)$ & $1.2(2)$ & $3.5(3)$ & $2.3(4)$ \\
$\mathrm{Ni}-20 \mathrm{Re}$ & $3.2(2)$ & $0.9(2)$ & $2.3(2)$ & $0.8(3)$ \\
$\mathrm{Ni}-20 \mathrm{Ta}$ & $3.5(1)$ & $1.2(2)$ & $2.9(2)$ & $0.9(4)$ \\
$\mathrm{Ni}-20 \mathrm{~W}$ & $3.2(1)$ & $1.1(2)$ & $2.5(3)$ & $1.1(2)$ \\
\hline
\end{tabular}

simulations of $\mathrm{Ni}$ and binary $\mathrm{Ni}_{400} \mathrm{X}_{100}(\mathrm{X}=\mathrm{Al}, \mathrm{W}, \mathrm{Ta}, \mathrm{Re})$ liquids at $1830 \mathrm{~K}$. These results are from the lowest pressure $\mathrm{nVT}$ simulations for that alloy composition. A linear fit to the curves yields a slope that can be used to estimate the concentration dependent tracer diffusion constants at this temperature. Similar to previous AIMD simulations, these data exhibit an initial ballistic trajectory before producing diffusive motion [15]. The AIMD simulations produce an initial ballistic regime of approximately $0.3 \mathrm{ps}$, and produce errors smaller than the statistical errors in the final diffusion rates. Also, all the simulations undergo an initial thermalization period, typically $\sim 0.1 \mathrm{ps}$, before calculating thermal averages. The predicted diffusion parameters for these alloys are shown in Table I. These are derived from mean square displacements at three or four volumes with linear fits of diffusion constants as a function
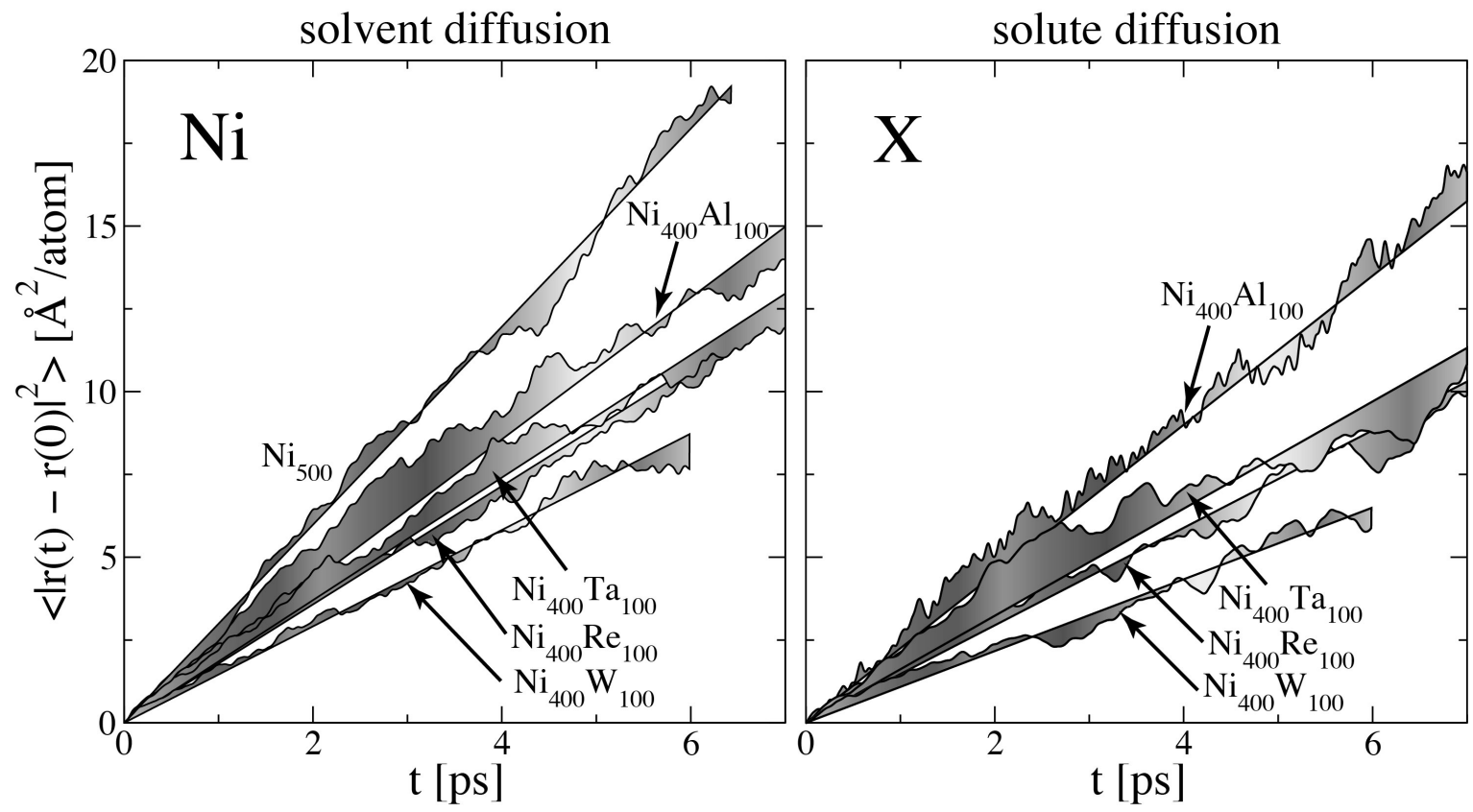

Figure 2. Solvent and solute mean squared displacements in $\mathrm{Ni}$ and $\mathrm{N}-20 \mathrm{X}(\mathrm{x}=\mathrm{Al}, \mathrm{Re}, \mathrm{Ta}, \mathrm{W})(\mathrm{at} \%)$ at $1830 \mathrm{~K}$. Slopes of these curves are used with the Einstein relation to derive the diffusion constant for these atomic species. 
of volume to derive the zero pressure diffusion rates. Slopes of these curves are also used to derive the activation volume at the equilibrium volume using the definition:

$\mathrm{V}_{\mathrm{a}}=-\mathrm{k}_{\mathrm{B}} \mathrm{Td}(\ln \mathrm{D}) / \mathrm{dP}$

Diffusion results for $\mathrm{Ni}$ are in good agreement with previous AIMD simulations of Jask and co-workers who reported self diffusion of $4.4 \times 10^{-5} \mathrm{~cm}^{2} / \mathrm{sec}$ at $1850 \mathrm{~K}$ [16]. Current results predict Re and $\mathrm{Ta}$ as the slowest solute diffusion species, a result consistent with their atomic mass and size. $\mathrm{Ni}$ diffusion is slower in the binary melts, an effect that increases with solute concentration. From this limited data this effect appears roughly linear with solute concentration, even though the average atomic volume is increasing. This is consistent with strong solventsolute interactions, as found in the previous section.

Due to the lack of radio isotopes for $\mathrm{Ni}$ classical measurements of self-diffusion are problematic. However there have been some recent inelastic neutron scattering experiments on $\mathrm{Al}$ rich $\mathrm{Ni}$-Al melts. Using these methods for alloys at $1995 \mathrm{~K}$ Chathoth and coworkers measured the self-diffusion of $\mathrm{Ni}$ at $3.8 \times 10^{-5}$ $\mathrm{cm}^{2} / \mathrm{sec}$ and for Ni-25Al (at\%) a self-diffusion of $3.9 \times 10^{-5}$ $\mathrm{cm}^{2} / \mathrm{sec}$ [17, 18]. This is in excellent agreement with our calculated values for $\mathrm{Ni}$ self-diffusion for simple metal $\mathrm{Ni}$ and $\mathrm{Ni}-20 \mathrm{Al}$ at $1830 \mathrm{~K}, 5.3(3) \times 10^{-5} \mathrm{~cm}^{2} / \mathrm{sec}$ and $3.4(4) \times 10^{-5}$ $\mathrm{cm}^{2} / \mathrm{sec}$ respectively. Also there are some limited solute tracer diffusion measurements in $\mathrm{Ni}-2 \mathrm{~W}$ (at $\%$ ), which observed a diffusion coefficient of $2.4(2) \times 10^{-5} \mathrm{~cm}^{2} / \mathrm{sec}$ for $\mathrm{W}$ in the temperature range of 1755-2022 K [18]. Again this is in good agreement with our results for Ni-5.4W (at\%) at $1830 \mathrm{~K}$ where we find solute diffusion rate of $3.6(4) \times 10^{-5} \mathrm{~cm}^{2} / \mathrm{sec}$.

The good statistics for the diffusion constants, and Figure 2, illustrate that significant interdiffusion in occurring over the timescales of these molecular dynamic simulations. These results show that AIMD simulations run for approximately $7 \mathrm{ps}$ have evolved over a sufficiently long period of time to produce reasonable estimates of the equilibrium molar volumes.

Molar Volumes in $\mathrm{Ni}$, binary and ternary alloys:

Initial analysis of the molar volumes centers on documenting the accuracy and precision of the AIMD method as applied to simple, binary and ternary Ni alloy liquid-metal-densities. With this established, attention then turned to predicting density inversion in ternary and $\mathrm{Ni}$-base alloys.

The first nVT dynamics calculations of molar volumes focused on simple metal, binary and ternary Ni alloys. Here a series of constant volume calculations were used to find the pressure as a function of volume. The time average pressures were then fit to a second order polynomial to derive an equation of state. The equation of state yields the equilibrium volume $\left(\mathrm{V}_{0}\right)$, bulk modulus $\left[\mathrm{B}=-\mathrm{V}_{0}(\mathrm{dP} / \mathrm{dV})\right]$, and its pressure derivative. Table II shows the predicted molar volumes, with expected error, with the corresponding parametric result from Mukai and coworkers, produced from fits to experimental measurement [2]. Errors are estimated using standard error propagation and correlation times of $50 \mathrm{fs}$ [19]. A more detailed description of this analysis, numerical results for $\mathrm{B}$, and $\mathrm{B}$ ', and how these results can be used to estimate other quantities can be found in the literature [11].

The calculated molar volumes are in good agreement sessile drop experiments, for elemental $\mathrm{Ni}$ at these temperatures the error is $1.6 \%$ and $0.8 \%$ for $1750 \mathrm{~K}$ and $1830 \mathrm{~K}$, respectively [2]. When the (modified) sessile drop and (modified) pycnometric measurements of Mukai and coworkers are used to interpolate densities for $\mathrm{Ni}-19.7 \mathrm{Al}$ and $\mathrm{Ni}-5.83 \mathrm{~W}$ (at. \%) we find average errors in the AIMD results to be below 1.8\%. There are also several other recent measurements of liquid $\mathrm{Ni}$ alloy densities. For example, Feng et al. measured the density of elemental Ni and dilute solutions of $\mathrm{W}$ in Ni using a sessile drop method [20]. The AIMD predictions are within $1.4 \%$ of these elemental $\mathrm{Ni}$ results. Since Ni at these temperatures is chemically reactive it is also worth considering non-contact methods, where the liquid metal is not in contact with a physical plate or containment vessel. One such noncontact technique, developed by Plevachuk and co-workers, uses electromagnetic levitation and optical dilatometry to measure the high temperature liquid metal densities [21]. The AIMD predictions for $\mathrm{Ni}-25 \mathrm{Al}$ are within $0.6 \%$ of values measured using these methods. In order to illustrate the trend in the molar volume data the AIMD and Mukai results are plotted in Figure 3. For compositions inside the range of Mukai's experimental database the agreement between the two methods is within $2 \%$, as indicated by the dotted lines. However, when the model needs to extrapolate to the AIMD sampled compositions errors can be quite large (i.e. $\mathrm{Ni}_{400} \mathrm{~W}_{100}, \mathrm{Ni}_{473} \mathrm{Ta}_{27}$, and $\mathrm{Ni}_{400} \mathrm{Re}_{100}$ ). Also for the case of $\mathrm{Ni}_{400} \mathrm{Ta}_{100}$, not shown in Fig. 3, the Mukai parameterization diverges from the AIMD result by at least $7 \mathrm{~cm}^{3} /$ mole. There is also a systematic increase in the difference between the fitted and AIMD results with decreasing temperature; this is an indication that the two methods produce different coefficients of thermal expansion.

Table II. Calculated atomic volumes $\left(\mathrm{cm}^{3} /\right.$ mole $)$ from current AIMD simulations and the Mukai parameterization based on extensive experiments on binary alloys [2].

\begin{tabular}{|l|l|l|l|l|}
\hline \multirow{2}{*}{ Composition } & \multicolumn{2}{|c|}{$\mathrm{T}=1750 \mathrm{~K}$} & \multicolumn{2}{c|}{$\mathrm{T}=1830 \mathrm{~K}$} \\
\cline { 2 - 5 } $\mathrm{Al}$ & AIMD & Mukai & AIMD & Mukai \\
$\mathrm{Ni}$ & $12.80(2)$ & & $12.91(3)$ & \\
$\mathrm{Ni}-20 \mathrm{Al}$ & $7.57(1)$ & 7.460 & $7.62(1)$ & 7.572 \\
$\mathrm{~N}-5.4 \mathrm{~W}$ & $7.88(1)$ & 7.763 & $7.94(1)$ & 7.849 \\
$\mathrm{Ni}-20 \mathrm{~W}$ & $7.66(1)$ & 7.553 & $7.70(1)$ & 7.652 \\
$\mathrm{Ni}-5.4 \mathrm{Re}$ & $7.94(1)$ & 6.519 & $7.98(1)$ & 7.329 \\
$\mathrm{Ni}-20 \mathrm{Re}$ & $7.65(1)$ & 7.494 & $7.69(1)$ & 7.650 \\
$\mathrm{Ni}-5.4 \mathrm{Ta}$ & $7.91(1)$ & 7.587 & $7.93(1)$ & 7.859 \\
$\mathrm{Ni}-20 \mathrm{Ta}$ & $7.70(1)$ & 7.331 & $7.75(1)$ & 7.505 \\
$\mathrm{Ni}-10 \mathrm{Al}-2.8 \mathrm{~W}$ & $8.14(1)$ & --- & $8.18(1)$ & -- \\
$\mathrm{Ni}-10 \mathrm{Al}-2.8 \mathrm{Re}$ & $7.77(1)$ & 7.692 & $7.80(1)$ & 7.766 \\
$\mathrm{Ni}-10 \mathrm{Al}-2.8 \mathrm{Ta}$ & $7.79(1)$ & 7.629 & $7.80(1)$ & 7.751 \\
\hline
\end{tabular}



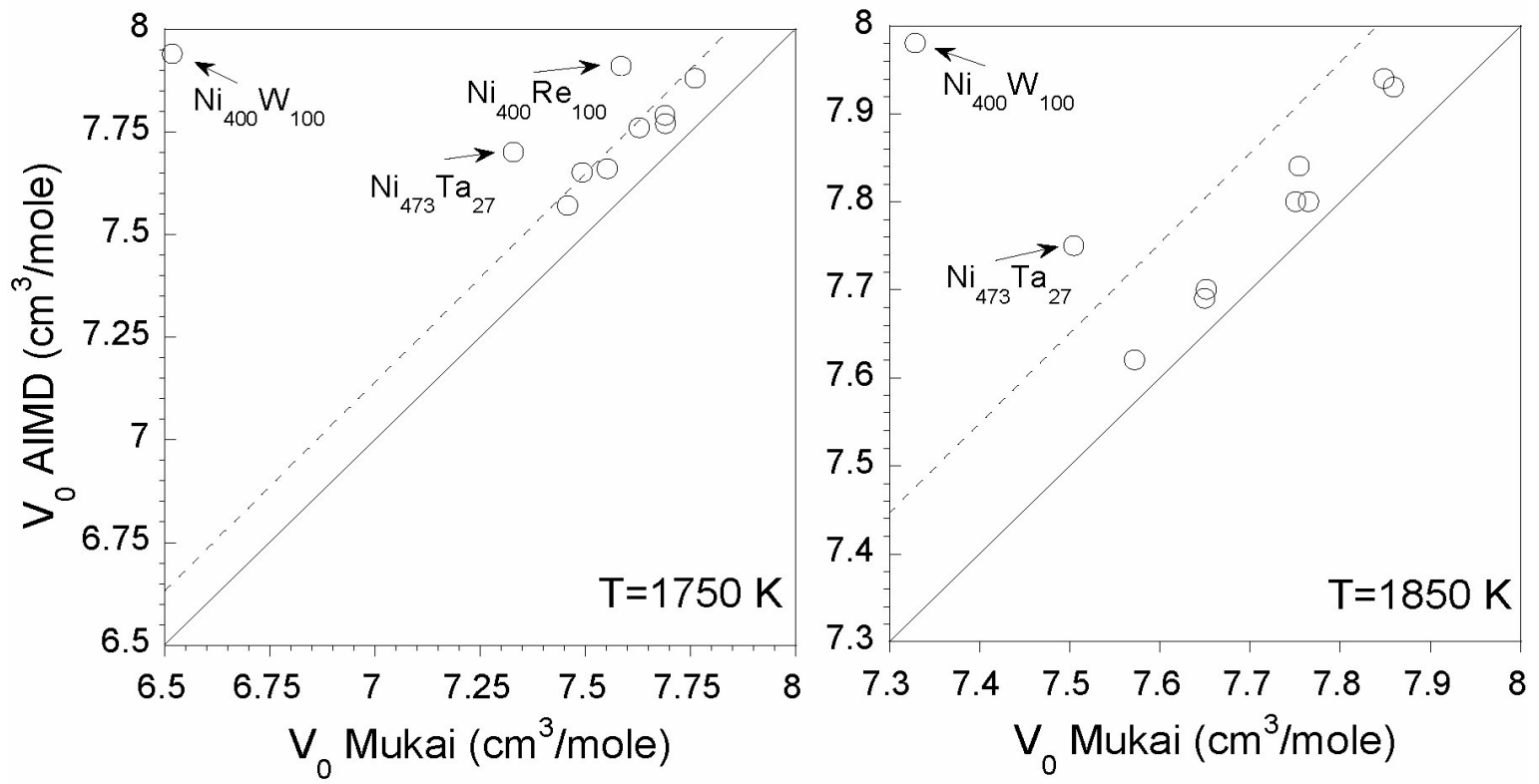

Figure 3. Comparison of molar volumes calculated with AIMD simulations and estimated using the Mukai parameterization [2]. Data in perfect agreement would fall on the line representing a slope of one, the dashed line indicates where the two results differ by $2 \%$.

The outliers are compositions that fall outside the composition ranges included in the fit of the Mukai model.

The ternary alloy compositions, in table II, were chosen in part to assess the accuracy of interpolating the molar volumes in complex alloys based on measured values in binary alloys. For example the Mukai parameterization implicitly assumes that the complex alloy molar volume can be approximated by the sum of partial molar volumes of non-interacting chemical species. Figure 4 illustrates a path in the ternary triangle using the available compositions in table II. The ternary alloys, $\mathrm{Ni}_{436} \mathrm{Al}_{50} \mathrm{X}_{14}$, can be expressed as the average of the molar volumes fro $\mathrm{Ni}_{400} \mathrm{Al}_{100}$ and $\mathrm{Ni}_{473} \mathrm{X}_{27}$, where $\mathrm{X}=\mathrm{W}, \mathrm{Re}$, or Ta. Deviations from linearity, in Figure 4 , would indicate a breakdown in the assumption of expressing the complex molar volume as a linear combination of binary molar volumes. Only in the case of Ni473Ta27, using the Mukai parameterization is the deviation from linearity significant $(\sim 3 \%)$. This is most likely a reflection of forcing the method to extrapolate the molar volume outside of the range of reference binary compositions (e.g extrapolation). In most cases the deviation from linearity is less than $0.4 \%$ using the Mukai model validating the underlying assumptions.

The temperature dependence of the molar volumes can be calculated from the AIMD results in several ways. Liquid density measurements in these alloys show linear temperature dependence, so finite difference should be a reasonable approximation. An alternative method that uses the liquid bulk modulus and the ensemble averaged pressure is reviewed in the literature [11]. Figure 5 shows representative AIMD predications for the thermal expansion coefficient in $\mathrm{Ni}$ and $\mathrm{Ni}$ $5.4 \mathrm{~W}$ (at \%) for the temperature range 1750-1950 K. Here both NVT and NPT dynamics were used to assess the molar volume as a function of temperature yielding volumetric expansion coefficients of $8.5 \times 10^{-5} \mathrm{~K}^{-1}$ and $6.7 \times 10^{-5} \mathrm{~K}^{-1}$ for $\mathrm{Ni}-5.4 \mathrm{~W}$ and $\mathrm{Ni}$ respectively. Comparing directly to the work of Mukai we find that the AIMD predictions are consistently lower than the sessile drop and pycnometric measurements. For the alloys shown in Table II agreement is at best within a factor of two. A wider variety of methods have been used to measure $\beta$ in $\mathrm{Ni}$, producing results ranging from $1.0-1.5 \times 10^{-4} \mathrm{~K}^{-1}[22,23]$. Most of these techniques are contact methods, using a plate or accurately machined crucible. In sessile drop experiments a drop of liquid metal is in contact with a solid surface and the shape of the drop is used to estimate the liquid metal density. Other methods may be preferred for the Ni alloys, where surface tension may be influenced by possible liquid contamination from the substrate or crucible. One earlier study, based on gamma ray attenuation, sidesteps possible bias in the surface tension and found a coefficient of thermal expansion for elemental liquid Ni of $9.42 \times 10^{-5} \mathrm{~K}^{-1}$ [24]. The only noncontact method density measurement technique that has been applied to the $\mathrm{Ni}$ alloys is the very recent work of Plevachuk et al. where using electromagnetic levitation and optical dilatometry they find a coefficient of thermal expansion of $1.2 \times 10^{-4} \mathrm{~K}^{-1}$ for Ni$25 \mathrm{Al}$ (at.\%) [22]. Mukai's most recent work in Ni-Al alloys, using a modified sessile drop method, estimates $\beta$ of this alloy to be $1.3 \times 10^{4} \mathrm{~K}^{-1}$ [25]. In general the AIMD results are in better agreement with experiments that avoid possible bias in surface tension produced by chemical contamination from a crucible or substrate. These include the gamma ray attenuation and electromagnetic levitation/optical dilatometry techniques. However, even these show the AIMD computational method 


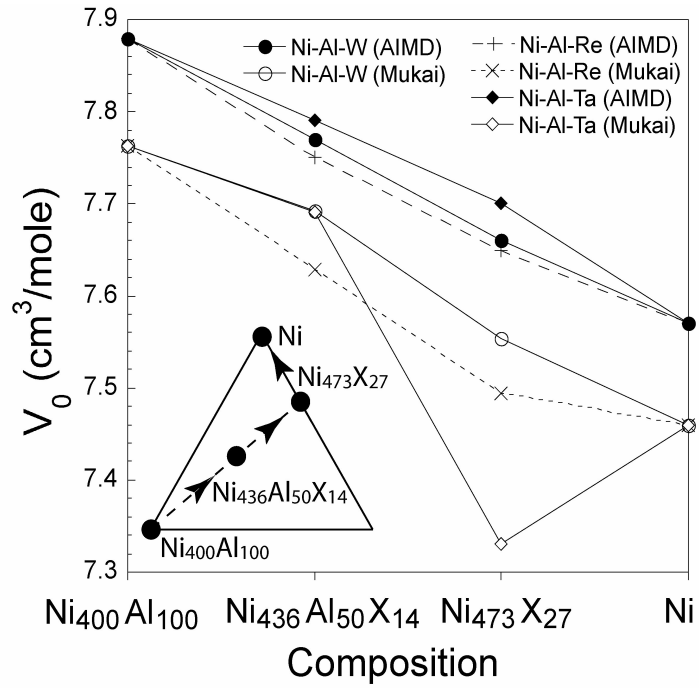

Figure 4. Comparison of molar volumes at $1750 \mathrm{~K}$ for $\mathrm{Ni}-\mathrm{Al}-\mathrm{X}$ $(\mathrm{X}=\mathrm{W}, \mathrm{Re}$, and $\mathrm{Ta})$ in the ternary triangle. With the exception of $\mathrm{Ni}_{473} \mathrm{Ta}_{27}$, deviations from linearity for most of the AIMD and Mukai [2] results are less than 0.8 and $0.4 \%$ respectively.

underestimates liquid metal coefficient of thermal expansion by approximately $25 \%$ for elemental $\mathrm{Ni}$.

Calculations for Density Inversion

Three alloys are considered for possible density inversion, $\mathrm{Ni}$ Al-W, CMSX-4, and Rene-N4. For the more complicated alloys it is first necessary to demonstrated that the 500 atom cell AIMD simulations can give accurate and reproducible densities for simulations running for less than $\sim 10$ ps. To demonstrate this four instantiations of Rene-N4, based on different random initial atomic distributions, were run at the solidus and liquidus temperatures for compositions determined from the Scheil solidification path, estimated using CalPhad (Pandat $\AA$ ) methods. The predicted compositions are Ni62.2-Cr11.0-Co5.2Mo1.3W1.9-A110.-Ti5.8-Ta2.3-Nb0.5 and Ni63.0-Cr11.2-Co7.6Mo0.9-W1.9-Al9.2-Ti4.3-Ta1.6-Nb0.3 at $1,635 \mathrm{~K}$ and $1,582 \mathrm{~K}$ respectively. The very dilute $\mathrm{Nb}$ additions were removed from the calculations with the balance in composition being spread proportionally overall the remaining species yielding the 500 atom cell compositions: $\mathrm{Ni}_{315} \mathrm{Cr}_{56} \mathrm{Co}_{38} \mathrm{Mo}_{5} \mathrm{~W}_{10} \mathrm{Al}_{46} \mathrm{Ti}_{22} \mathrm{Ta}_{8}$ and $\mathrm{Ni}_{312} \mathrm{Cr}_{55} \mathrm{Co}_{26} \mathrm{Mo}_{6} \mathrm{~W}_{10} \mathrm{Al}_{50} \mathrm{Ti}_{29} \mathrm{Ta}_{12}$ for $1,635 \mathrm{~K}$ and $1,582 \mathrm{~K}$ respectively. These chemistries were then used to construct 500 atom cells with random distributions of the appropriate elements. The different instantiations at each temperature were run in order to assess the influence of configurational entropy on the molar volume or alloy density. After $8 \mathrm{ps}$ the derived time averaged densities of the four cases at each temperature were found to be within statistical error, illustrating the efficacy of this approach. For this range of compositions and temperatures we conclude that a random configuration is a reasonable approximation of the melt and will give reproducible liquid metal densities.

For the model Ni-Al-W alloy we adopted a nominal composition of $\mathrm{Ni}-14 \mathrm{Al}-3 \mathrm{~W}$ (at\%) and evaluated the equilibrium liquid

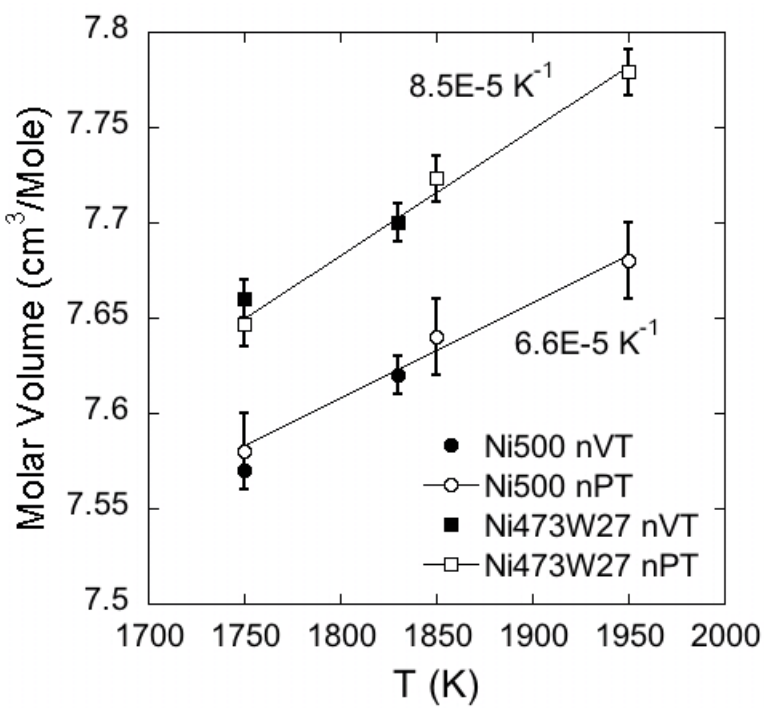

Figure 5. Variation in molar volume with temperature for elemental $\mathrm{Ni}$ and $\mathrm{Ni}_{473} \mathrm{~W}_{27}$ calculated using both $\mathrm{nVT}$ and $\mathrm{nPT}$ dynamics. The predicted coefficient of thermal expansion, $\beta$, is shown for both alloys.

compositions and temperatures for 0.5 and 1.0 liquid fraction (wt\%) using the Scheil model. This alloy was chosen because it has been demonstrated to produce freckle defects in conventional Bridgeman directional solidification. The predicted liquid compositions at 1711 and $1720 \mathrm{~K}$ are shown in Table III. The AIMD calculations predict a $2 \%$ increase in the density going from 1,711 to $1,720 \mathrm{~K}$, reflecting a relatively strong density inversion. The Scheil model predicts a change in composition that favors an increase in density at the higher temperature. However for this system there is a decrease in the molar volume with increasing temperature, from 7.782(5) to $7.767(8) \mathrm{cm}^{3} / \mathrm{mole}$, so density inversion is predicted even when the increase in elemental mass is not taken into account. When applied to this model alloy, the Mukai parameterization produces a large density inversion at these temperatures and chemistries (Table III). The estimated molar volumes are 7.7367 and 7.7302 for 1711 and $1720 \mathrm{~K}$ respectively, which in consistent with a small density inversion based on just volumetric effects within the Mukai parameterization.

Temperatures and compositions were also assessed from Sheil calculations for 0.6 and 1.0 liquid fraction (wt\%) for Rene-N4 and CMSX-4. Compositions for these temperatures and alloys

Table III. Predicted liquid metal densities for the Ni-Al-W model system. Here $\mathrm{f}_{\mathrm{L}}$ is the fraction liquid (wt\%) and the numbers in parenthesis indicate the $95 \%$ confidence level in the last digit.

\begin{tabular}{|c|c|c|c|c|}
\hline & & Composition & \multicolumn{2}{|c|}{ Density $\left(\mathrm{g} / \mathrm{cm}^{3}\right)$} \\
\hline $\mathrm{f}_{\mathrm{L}}$ & $\mathrm{T}(\mathrm{K})$ & Atomic $(/ 500)$ & AIMD & Mukai \\
\hline 1.0 & 1720 & $\mathrm{Ni}_{415} \mathrm{Al}_{70} \mathrm{~W}_{15}$ & $7.46(2)$ & 7.5040 \\
0.5 & 1711 & $\mathrm{Ni}_{410} \mathrm{Al}_{78} \mathrm{~W}_{12}$ & $7.29(1)$ & 7.3352 \\
\hline
\end{tabular}


Table IV. The compositions used in the AIMD calculations for Rene-N4 and CMSX-4 at 0.6 and 1.0 liquid fraction (wt\%). Both the atomic percent and the number of atoms in the simulation cell are given for each atomic species.

\begin{tabular}{|c|c|c|c|c|c|c|c|c|c|c|c|}
\hline Alloy & $\begin{array}{l}\text { Liquid } \\
\text { fraction }\end{array}$ & Conc. & $\mathrm{Ni}$ & Co & $\mathrm{Cr}$ & Mo & W & $\operatorname{Re}$ & $\mathrm{Al}$ & $\mathrm{Ta}$ & $\mathrm{Ti}$ \\
\hline \multirow{4}{*}{ Rene-N4 } & $0.6 \mathrm{f}_{\mathrm{L}}$ & at $\%$ & 62.5 & 7.2 & 11.3 & 1.0 & 1.7 & 0.0 & 9.4 & 2.0 & 4.9 \\
\hline & $\mathrm{T}=1604 \mathrm{~K}$ & \# & 312 & 36 & 57 & 5 & 8 & 0 & 47 & 10 & 25 \\
\hline & $1 \mathrm{f}_{\mathrm{L}}$ & at $\%$ & 63.3 & 7.5 & 11.2 & 0.9 & 1.9 & 0.0 & 9.2 & 1.6 & 4.3 \\
\hline & $\mathrm{T}=1622 \mathrm{~K}$ & \# & 316 & 37 & 56 & 5 & 10 & 0 & 46 & 8 & 22 \\
\hline \multirow{4}{*}{ CMSX-4 } & $0.6 \mathrm{f}_{\mathrm{L}}$ & at $\%$ & 63.2 & 8.8 & 7.7 & 0.4 & 1.8 & 0.7 & 13.2 & 2.8 & 1.4 \\
\hline & $\mathrm{T}=1635 \mathrm{~K}$ & \# & 316 & 44 & 38 & 2 & 9 & 4 & 66 & 14 & 7 \\
\hline & $1 \mathrm{f}_{\mathrm{L}}$ & at $\%$ & 63.8 & 9.3 & 7.6 & 0.4 & 2.0 & 1.0 & 12.6 & 2.2 & 1.3 \\
\hline & $\mathrm{T}=1654 \mathrm{~K}$ & \# & 319 & 46 & 38 & 2 & 10 & 5 & 63 & 11 & 6 \\
\hline
\end{tabular}

are shown in Table IV. Results for all these calculations are shown in Figure 6. AIMD predictions for both Rene-N4 and CMSX-4 suggest density inversions under these conditions, though they are smaller than that seen in Ni-Al-W. Note that two instantiations were run for each temperature-compositions for these superalloys. Also, the increase in density for CMSX-4 is approaching the statistical error of the predictions. The Mukai parameterization does not predict a density inversion for CMSX4 these compositions and temperatures. However, the DFT predictions and the Mukai parameterization for the Ni-Al-W alloys are very similar in both absolute size and in the degree of density inversion. Finally, both methods predict a density inversion for Rene-N4 at these temperatures and compositions. For Rene-N4 the molar volume also reflects a density inversion for both the AIMD and Mukai parameterization.

Given the different results for CMSX-4, and the shallow gradient found in the AIMD calculations for this alloy, further calculations were undertaken to better describe the change in density in the mushy zone. A series of calculations were

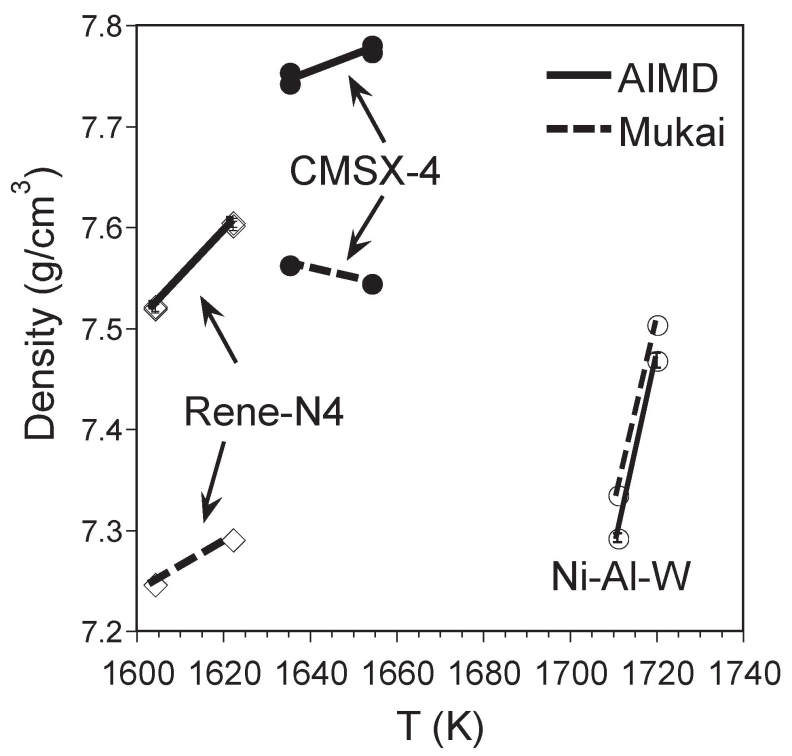

Figure 6. Density as a function of temperature for Ni-Al-W, Rene-N4 and CMSX-4 for 0.6 and 1.0 liquid fraction (wt\%). performed for liquid volume fractions ranging from the expected liquidus and solidus. These results, shown in Figure 7, illustrate the expected trends in liquid metal density during solidification. The additional data points suggest that within statistical error CMSX-4 is predicted to sample density inversion between 0.4 and 1.0 liquid fraction in the melt. The other two alloys also shown in Figure 7 to illustrate the relative strength of the density inversion. Finally, as shown in Eq. 1, another measure of the influence of the density inversion is the density contrast over the mushy zone. This is the dimensionless quantity $\Delta \rho / \rho$ which we define as $\left(\rho_{0}-\rho(h)\right) / \rho_{0}$, where $\rho_{0}$ is the density at the liquidus and $\rho(h)$ is the density at a specific height in the mushy zone. In this case we use the values of the density at 0.6 liquid fraction (wt $\%$ ) for $\rho(h)$. Results for the three alloys are shown in Table V. While the AIMD predictions for the density contrast of CMSX-4 have the opposite sign of the Mukai predictions (consistent with a density inversion) the rank ordering of density contrast is consistent between the two methods. Also, it is worth noting that the rank ordering of the density contrast is in agreement with the predictions of Reed and coworkers, based on

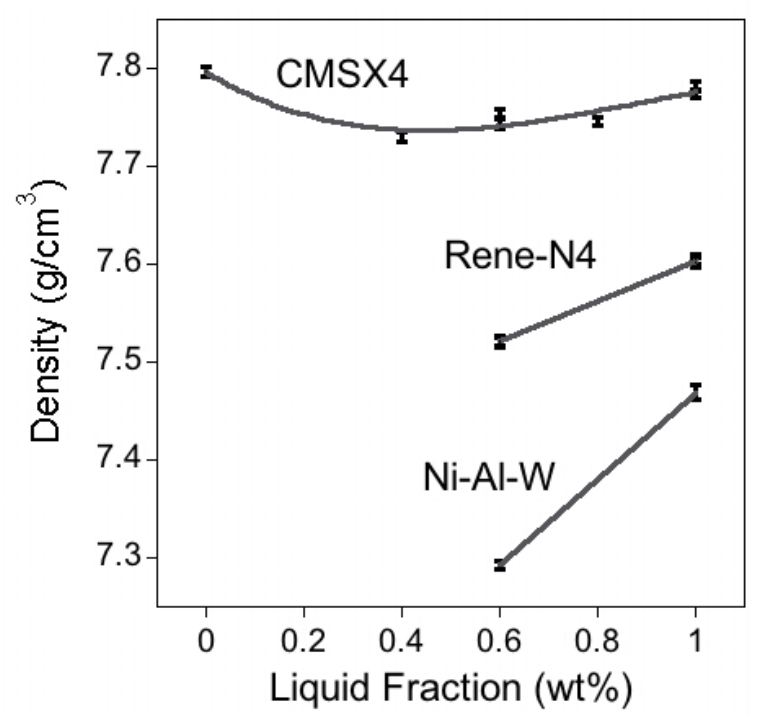

Figure 7. Calculated liquid metal densities for CMSX-4, Rene$\mathrm{N} 4$ and a model Ni-Al-W alloy for a range of liquid fraction (wt \%). 
Table V. Predicted density contrast $(\Delta \rho / \rho)$, based on the liquidus and 0.6 liquid fraction $(\mathrm{wt} \%)$ densities.

\begin{tabular}{|c|c|c|c|}
\hline Method & CMSX-4 & Rene-N4 & Ni-Al-W \\
\hline AIMD & 0.00373 & 0.01085 & 0.02355 \\
Mukai [2] & -0.00245 & 0.00609 & 0.02251 \\
\hline
\end{tabular}

ThermoCalc predictions (for chemistry) across the height of the mushy zone [26].

\section{Summary}

AIMD simulations have been performed to derive the timeaveraged properties of liquid metals for alloys ranging from simple metals to Ni-based superalloys such as Rene-N4 and CMSX-4. For the simpler alloys diffusion rates are in good agreement with available experimental measurements, and the radial distribution functions show signs of short range ordering consistent with $\mathrm{Ni}$ being surrounded by unlike neighbors. Results show that the AIMD method is sampling an adequate volume and time domain. Predicted molar volumes for elemental, binary and ternary Ni alloys are typically within $\sim 2 \%$ of measured values and but underestimate the coefficients of thermal expansion as compared to experimental measurements using contact methods. The calculations confirm the assumption that the molar volumes of complex alloys can be described using a linear combination of results from simpler binary alloys.

Densities of three freckle prone alloys, Ni-Al-W, Rene-N4 and CMSX-4, were studied for compositions and temperatures expected in the mushy zone. Calculations for the model ternary Ni-Al-W alloy predict a $2 \%$ density inversion, consistent with convective instabilities that produce freckle defects. Simulations of reference Rene-N4 alloys, based on random distributions of atomic species, were used to illustrate the precision and reproducibility of molar volume predictions using a 500 atom cell. Calculations for Rene-N4 and CMSX-4 also produce density inversions, though smaller than that found for the model Ni-Al-W alloy. The Mukai parameterization produced the same ranking in terms of the size of density contrast for these three alloys. However, CMSX-4 is not predicted to have a density inversion in contrast with the AIMD results. Overall, these results suggest that even for highly complex chemistries moderately sized AIMD simulations can produce reliable results. With proper validation this approach could be used to assess convection instabilities in a wide range of materials. Similar methods can be used to predict other parameters used in the Rayleigh-number criteria, such as the shear viscosity, that are difficult to measure and are not well known for complex alloys.

\section{Acknowledgements}

This research was supported in part by a grant of computer time from the DoD High Performance Computing Modernization Program at the Air Force Research Laboratory DoD Supercomputing Research Center AFRL-DSRC. MA and DRT were supported in part with a grant from the Air Force Office of Scientific Research under the Materials Engineering for Affordable New Systems (MEANS-II) program. The authors gratefully acknowledge insightful discussions with T. Pollock, P. Voorhees, J. Miller, O. Senkov, and S.H. Davis, and the technical assistance provided by S. Kajihara at the AFRLDSRC.

\section{References}

[1] C. Beckermann, J.P. Gu, and W.J. Boettinger, "Development of a freckle predictor via Rayleigh number method for single-crystal Nickel-base superalloy castings", Met. Trans. A, 31A (2000) 2545.

[2] K. Mukai, Z.S. Li, and K.C. Mills, "Prediction of the densities of liquid Ni-based superalloys", Met. Mater. Trans. B, 36 (2005) 255.

[3] G. Kresse, and J. Hafner, "Efficiency of ab-initio total energy calculations for metals and semiconductors using a plane-wave basis set”, Phys. Rev. B, 49 (1994) 14251; Kresse, G. and J. Furthmüller, J. Computat. Mater. Sci., 6 (1996) 15.

[4] J. P. Perdew and Y. Wang, "Accurate and simple analytic representation of the electron-gas correlation energy", Phys. Rev. B, 45 (1992) 13244.

[5] D. Vanderbilt, "Soft self-consistent pseudopotentials in a generalized eigenvalue formalism", Phys. Rev. B, 41 (1990) 7892.

[6] G. Kresse and D. Joubert, "From ultrasoft pseudopotentials to the projector augmented-wave method", Phys. Rev. B 59 (1999) 1758.

[7] M. Asta, D. Morgan, J. J. Hoyt, B. Sadigh, J. D. Altho , D. de Fontaine, and S. M. Foiles, "Embedded-atom-method study of structural, thermodynamic, and atomic-transport properties of liquid Ni-Al alloys", Phys. Rev. B, 59 (1999) 14271.

[8] P.E. Blochl, "Projector augmented-wave method", Phys. Rev. B, 50 (1994) 17953.

[9] S. K. Das, J. Horbach, M. M. Koza, S. M. Chatoth, and A. Meyer, "Influence of chemical short-range order on atomic diffusion in Al-Ni melts", Appl. Phys. Lett., 86 (2005) 011918.

[10] L. Rosso, P. Minary, Z. Zhu, and M.E. Tuckerman, "On the use of the adiabatic molecular dynamics technique in the calculation of free energy profiles", J. Chem. Phys., 116 (2002) 4389

[11] C. Woodward, M. Asta, D.R. Trinkle, J. Lill, and S Angioletti-Uberti, "Ab-initio simulations of molten $\mathrm{Ni}$ alloys”, J. App. Phys., 107 (2010), 113522.

[12] G. W. Lee, A. K. Gangopadhyay, K. F. Kelton, R. W. Hyers, T. J. Rathz, J. R. Rogers, and D. S. Robinson, "Difference in icosahedral short-range order in early and late transition metal liquids", Phys. Rev. Lett., 93 (2004) 037802 . 
[13] W. K. Luo, H. W. Sheng, F. M. Alamgir, J. M. Bai, J. H. $\mathrm{He}$, and E. Ma, "Icosahedral short-range order in amorphous alloys", Phys. Rev. Lett., 92 (2004) 145502.

[14] F. Frank, "Supercooling of Liquids", Proc. R. Soc. London, Ser., A215 (1952) 43.

[15] X. Han, M. Chen, and Y. Lü, "A molecular dynamics study for the thermphysical properties of liquid Ti-Al alloys", Int. J. Thermophys., 29 (2008) 1408.

[16] N. Jakse, J. F. Wax, and A. Pasturel, "Transport properties of liquid nickel near the melting point: an ab-initio molecular dynamics study", J. Chem. Phys., 126 (2007) 234508 .

[17] S. M. Chathoth, A. Meyer, M. M. Koza, and F. Juranyi, "Atomic diffusion in liquid Ni, NiP, PdNiP, and PdNiCuP alloys", Appl. Phys. Lett., 85 (2004) 4881.

[18] J. P. Leonard, T. J. Renk, M. O. Thompson, and M. J. Aziz, "Solute diffusion in liquid nickel measured by pulsed ion beam melting”, Metall. Mater.Trans. A, 35 (2004) 2803.

[19] M.P Allen and D. J. Tildesley, "Computer Simulation of Liquids", (Oxford Science Publications, Clarendon Press, Oxford UK 1987).

[20] X. Feng, Y. Ren-Hui, F. Liang, L. Lan-Xiao, and Z. HongKai, "Densities of molten Ni-(Cr, Co, W) superalloys", Trans Nonferrous Met. Soc. China, 18 (2008) 24.

[21] Y. Plevachuk, I. Egry, J. Brillo, D. Holland-Moritz, and I. Kaban, "Density and atomic volume in liquid Al-Fe and Al-Ni binary alloys", Int. J. Mater. Res., 98 (2007) 107.

[22] T. Iida and R. Guthrie, "The Physical Properties of Liquid Metals”, (Clarendon Press, Oxford UK, 1988).

[23] A. F. Crawley, "Densities of liquid metals and alloys", Int. Metall. Rev., 19 (1974) 32.

[24] W. D. Drotning, in Thermal Expansion 7, edited by D. C. Larson, (Plenum, New York, 1988), 17-28.

[25] L. Fang, S. F. Zhang, F. Xiao, R. H. Yang, and K. Mukai, "Measurement and analysis of liquid density of $\mathrm{Ni}-\mathrm{Al}$ binary alloys", J. Alloys Compd. 493 (2010) 465.

[26] R.C. Reed, T. Tao, and N. Warnken, "Alloys by design: Application to nickel-based single crystal superalloys", Acta Mat., 57, (2009) 5898 -5913. 